\title{
Porous versus Compact Nanosized Fe(III)-Based Water Oxidation Catalyst for Photoanodes Functionalization
}

\author{
Michele Orlandi, ${ }^{* \dagger}{ }^{\dagger}$ Nicola Dalle Carbonare, ${ }^{\ddagger}$ Stefano Caramori, ${ }^{\ddagger}$ Carlo A. Bignozzi, ${ }^{\ddagger}$ Serena Berardi, ${ }^{\ddagger}$ \\ Alberto Mazzi, ${ }^{\dagger}$ Zakaria El Koura, ${ }^{\dagger}$ Nicola Bazzanella, ${ }^{\dagger}$ Nainesh Patel,${ }^{\S}$ and Antonio Miotello ${ }^{\dagger}$ \\ ${ }^{\dagger}$ Department of Physics, University of Trento, I-38123, Povo, Trento, Italy \\ ${ }^{\ddagger}$ Department of Chemical and Pharmaceutical Sciences, University of Ferrara, Via Fossato di Mortara 17-19, 44100 Ferrara, Italy \\ ${ }^{\S}$ Department of Physics, University of Mumbai, Vidyanagari, Santacruz (E), Mumbai 400 098, India
}

\section{Supporting Information}

\begin{abstract}
Integrated absorber/electrocatalyst schemes are increasingly adopted in the design of photoelectrodes for photoelectrochemical cells because they can take advantage of separately optimized components. Such schemes also lead to the emergence of novel challenges, among which parasitic light absorption and the nature of the absorber/catalyst junction features prominently. By taking advantage of the versatility of pulsed-laser deposition technique, we fabricated a porous iron(III) oxide nanoparticle-assembled coating that is both transparent to visible light and active as an electrocatalyst for water oxidation. Compared to a compact morphology, the porous catalyst used to functionalize crystalline hematite photoanodes exhibits a superior photoresponse, resulting in a drastic lowering of the photocurrent overpotential (about $200 \mathrm{mV}$ ) and a concomitant 5-fold increase in photocurrents at $1.23 \mathrm{~V}$ versus reversible hydrogen electrode. Photoelectrochemical impedance spectroscopy indicated a large increase in trapped surface hole capacitance coupled with a decreased charge transfer resistance, consistent with the possible formation of an adaptive junction between the absorber and the porous nanostructured catalyst. The observed effect is among the most prominent reported for the coupling of an electrocatalyst with a thin layer absorber.
\end{abstract}

KEYWORDS: pulsed-laser deposition, a-Fe $\mathrm{O}_{3}$, water splitting, photoanodes, hematite, adaptive junction, nanostructures

\section{INTRODUCTION}

Materials designed for photodriven fuel synthesis must perform two essential functions: (1) sunlight absorption to generate electron-hole pairs and (2) catalysis of the fuel-forming reactions. The latter becomes critical when multielectron redox reactions are involved, leading to slow kinetics and consequent loss of efficiency. A significant example is photochemical water electrolysis performed in a photoelectrochemical cell (PEC), where the strict kinetic requirements dictated in particular by the oxygen evolution reaction, which involves the protoncoupled exchange of four electrons, have driven for decades the search for efficient catalysts. ${ }^{1-3}$

In recent years, the research efforts have been increasingly directed toward schemes where the two functions are separately optimized and then integrated by functionalization of absorber materials with redox catalysts. Examples include inorganic semiconductors or molecular chromophores as absorbers, functionalized by coupling with molecular catalysts or nanostructures of metals or metal oxides. ${ }^{4-11}$ This kind of scheme allows for a simpler yet more in-depth screening of candidate catalysts while taking advantage of materials with already optimized optical properties (e.g., for photovoltaic applications) but also presents new challenges. Two critical issues are (a) the nature of the absorber/catalyst junction and (b) the parasitic light blocking ${ }^{5,12}$ by catalysts deposited on the absorber surface. The former depends largely on the morphology of the catalyst, with dense ion-impermeable structures yielding buried junctions and nonoptimal performance. By contrast, porous ion-permeable architectures can lead to adaptive junctions and substantially enhanced charge separation and photovoltage generation. ${ }^{13}$ As for the latter, in the case of metal oxides oxygen evolving catalysts (OEC) deposited on photoanodes, the effect of optical losses on the overall efficiency is so severe that in a recent investigation the best performance was obtained with a subnanometric film thickness. ${ }^{14}$ However, a higher catalyst mass loading is generally beneficial to reduce overpotential. This trade-off between optical losses and catalyst loading limits the application of earth-abundant, cheap, and scalable alternatives to noble-metalbased catalysts because the replacements generally have lower catalytic activity and higher quantities would be needed to produce a comparable performance. ${ }^{15,16}$

Received: April 29, 2016

Accepted: July 22, 2016

Published: July 22, 2016 
In this paper we address both issues by the design and fabrication of an amorphous $\mathrm{Fe}_{2} \mathrm{O}_{3}\left(\mathrm{a}-\mathrm{Fe}_{2} \mathrm{O}_{3}\right)$ OEC with a porous morphology consisting of a nanoparticle-assembled coating. The porous nature of the catalyst allows for ion permeability, leading to the probable formation of an adaptive junction with the photoactive material while maintaining high transparency in the visible region, likely due to the very small size of the nanometric structures $(\leq 5 \mathrm{~nm})$, possibly leading to a quantum confinement effect, as recently reported for hematite. ${ }^{17}$ Capitalizing on the versatility of pulsed-laser deposition (PLD), a physical deposition method that relies on laser ablation of a target material, we were able to perform a comparative investigation where by changing the deposition conditions different morphologies of a catalytic material having the same nominal composition (i.e., a largely amorphous iron(III) oxide), ranging from a dense compact layer to a porous nanoparticle-assembled coating, were cast on nanocrystalline hematite thin layers (HTL) acting as light absorber. The latter was selected as model host substrate by virtue of their ease of fabrication with good reproducibility and acceptable baseline performances in photoelectrochemical water oxidation arising from their thickness (ca. $30 \mathrm{~nm}$ ), which not only yields modest light-harvesting capability but also is compatible with the short carrier diffusion lengths of hematite. ${ }^{18}$ The photoresponse of these modified photoanodes, consisting of the integrated absorber-catalyst system, was largely affected by the specific nature of the catalytic layer. The dense film deposition invariably leads to a large decrease in the photoelectrochemical response with respect to the unmodified film, and photoanodes decorated with the porous catalyst show a drastic lowering of the photocurrent overpotential (about $200 \mathrm{mV}$ ) and a concomitant 5-fold increase in photocurrents at $1.23 \mathrm{~V}$ versus reversible hydrogen electrode (vs RHE) in $0.1 \mathrm{M} \mathrm{NaOH} \mathrm{(pH} \mathrm{13.3).} \mathrm{This} \mathrm{provides} \mathrm{an}$ additional guideline, besides the evaluation of the "dark" electrochemical activity of the oxygen evolving species, on the properties that should be pursued for the successful integration of a charge transfer catalyst in a photoelectrode for water splitting. Clearly, the choice of iron oxide as both the absorber material and OEC is motivated by the need to find earthabundant, cheap, and scalable materials to enable the large-scale application of solar water-splitting schemes. Iron oxide not only satisfies all these requisites ${ }^{19}$ but also is nontoxic and environmentally safe.

\section{EXPERIMENTAL METHODS}

Materials. $\mathrm{FeCl}_{3} \cdot 6 \mathrm{H}_{2} \mathrm{O}(\geq 99 \%)$ and $\mathrm{NaOH}$ (98\%) were purchased from Alfa Aesar. Polyethylene glycol bisphenol A epichlorohydrin copolymer (mol wt 15000-20 $000 \mathrm{Da}$ ) were purchased from SigmaAldrich. Fluorine-doped tin oxide glass slides (FTO, TEC $8 \Omega / \mathrm{cm}^{2}$, Hartford Glass) were cleaned by successive $30 \mathrm{~min}$ ultrasonic treatments in acetone, ethanol, aqueous Alconox (Sigma-Aldrich) solution, and distilled water and then blown dry with a warm air stream. A portion of the slides was then masked with KAPTON tape (Tesa) to preserve an uncovered zone for the electric contact. A metallic iron disc (purity 99.9\%, Sematrade) was used as the target material for PLD.

Crystalline Hematite Thin Layers (HTL) Preparation. $\mathrm{FeCl}_{3} \cdot 6 \mathrm{H}_{2} \mathrm{O}$ $(0.54 \mathrm{~g})$ was dissolved in DI water $(20 \mathrm{~mL})$, and then polyethylene glycol bisphenol A epichlorohydrin copolymer $(0.54 \mathrm{~g})$ was added under gentle stirring to avoid the formation of bubbles. The solution was spin-coated onto FTO glass (1000 rpm for $9 \mathrm{~s}$ and $2000 \mathrm{rpm}$ for $30 \mathrm{~s}$ ). After each spin-coated cycle the glass slide was annealed at 550 ${ }^{\circ} \mathrm{C}$ for $15 \mathrm{~min}$ in air. Spin-coating cycles were repeated 3 times, and after the third cycle, the electrode was heated at $550{ }^{\circ} \mathrm{C}$ for $2 \mathrm{~h}$ and ramped to $800{ }^{\circ} \mathrm{C}$ and held for $10 \mathrm{~min}$. The electrode was cooled to room temperature (RT) by suspending the heating.

Amorphous Iron Oxide $\left(a-\mathrm{Fe}_{2} \mathrm{O}_{3}\right)$ Pulsed Laser Deposition. a$\mathrm{Fe}_{2} \mathrm{O}_{3}$ was deposited on commercial (Sigma-Aldrich) quartz and ptype $\mathrm{Si}$ substrates (for UV-vis and scanning electron microscopy characterization, respectively) on FTO electrodes (for Raman spectroscopy and electrochemistry experiments) and on FTO/HTL electrodes (for photoelectrochemistry experiments) by the PLD technique using a $\mathrm{KrF}$ excimer laser (Lambda Physik LP 220i) with an operating wavelength of $248 \mathrm{~nm}$, pulse duration of $25 \mathrm{~ns}$, repetition rate of $20 \mathrm{~Hz}$, and laser fluence of $2.0 \mathrm{~J} / \mathrm{cm}^{2}$. The deposition chamber was evacuated up to a base pressure of $10^{-4} \mathrm{~Pa}$; then, oxygen gas was back-filled into the chamber through a mass flow controller. Deposition was performed at a constant oxygen pressure of $45 \mathrm{~Pa}$. The distance between the metallic iron target and substrates was set to $5.5 \mathrm{~cm}$ and the number of pulses was fixed (2000 pulses), to ensure that all samples have the same quantity of material. Substrate temperatures were either RT or $300{ }^{\circ} \mathrm{C}$ (DEP300). A more detailed description of the PLD apparatus is available elsewhere. ${ }^{20}$ For comparison, some samples were deposited at RT and then annealed at 300 and $550{ }^{\circ} \mathrm{C}$ in air in a tubular furnace. These are labeled AN300 and AN550 respectively. The heating rate was set to $10{ }^{\circ} \mathrm{C} / \mathrm{min}$, and the desired temperature was kept constant for $2 \mathrm{~h}$.

Characterization. Scanning electron microscopy (SEM) was used to analyze the morphology and the thickness of the films. Measurements were performed using a JEOL JSM-7001F FEG-SEM at $20.0 \mathrm{keV}$ electron beam energy. The SEM apparatus is equipped with an energy dispersive X-ray spectroscopy detector (EDXS, Oxford INCA PentaFETx 3 ). The working distance was maintained between 3 and $8 \mathrm{~mm}$. Surface morphology images were acquired in top-down and tilted modes, whereas cross-sectional analysis was performed putting the films on a $90^{\circ}$ stub.

Raman measurements were performed on a Horiba LabAramis setup equipped with a $\mathrm{HeNe} 633 \mathrm{~nm}$ laser as source and a confocal microscope ( $100 \times$ objective) coupled to a $460 \mathrm{~mm}$ focal length CCDbased spectrograph equipped with a four interchangeable gratings turret. In the range between 450 and $850 \mathrm{~nm}$, the wavenumber accuracy is $1 \mathrm{~cm}^{-1}$ with a $1800 \mathrm{~L} / \mathrm{mm}$ grating. The laser power is 15 $\mathrm{mW}$, and the maximum spot size is $5 \mu \mathrm{m}$. An accumulation number of 10 and an exposure time of $7 \mathrm{~s}$ were employed for all measurements.

UV-vis absorption was studied using a Varian Cary 5000 spectrophotometer, and quartz substrate was also used to investigate the UV region.

Stationary emission spectra were measured with an Edinburgh Instruments FLS 920 spectrofluorimeter, equipped with a double emission monochromator by using an excitation bandwidth $(\Delta \lambda)$ of 7 $\mathrm{nm}$. The ferric oxide thin films (RT, AN300, and DEP300) deposited on quartz slides were placed in a dedicated film holder equipped with a micrometer stage in order to optimize the angle between the thin film, the excitation beam and the emitted light reaching the detector. The $\mathrm{S} / \mathrm{N}$ ratio was optimized by summing 10 subsequent scans with $1 \mathrm{~nm}$ wavelength step and a dwell time of $0.1 \mathrm{~s}$. Spectra were corrected for the photomultiplier (R928P-Hamamatsu) response by using a factorybuilt calibration file. Excitation was in the absorption manifold of the ferric oxide, either at 350 or $300 \mathrm{~nm}$. Two different types of cutoff filters (395 and $350 \mathrm{~nm}$ ) prevented scattered excitation light from reaching the phototubes.

XPS was performed using a PHI 5000 VersaProbe II equipped with a monochromatic $\mathrm{Al} \mathrm{K} \alpha(1486.6 \mathrm{eV}) \mathrm{X}$-ray source and a hemispherical analyzer. Electrical charge compensation was required to perform the XPS analysis. The sample surface was sputtered for $30 \mathrm{~s}$ in order to remove any surface oxidation.

$J-V$ and constant potential electrolysis experiments were carried out in a three-electrode configuration cell using a platinum electrode and a saturated calomel electrode (SCE) as counter and reference electrodes, respectively. The cell containing aqueous $\mathrm{NaOH}$ (either 0.1 $\mathrm{M}, \mathrm{pH}$ 13.3, or $1 \mathrm{M}, \mathrm{pH} 14$ ) as electrolyte was connected to an Eco Chemie Autolab PGSTAT 302/N electrochemical work-station. J-V curves were recorded at a scan speed of $20 \mathrm{mV} \mathrm{s}^{-1}$. For the measurements under illumination, the working electrode was 

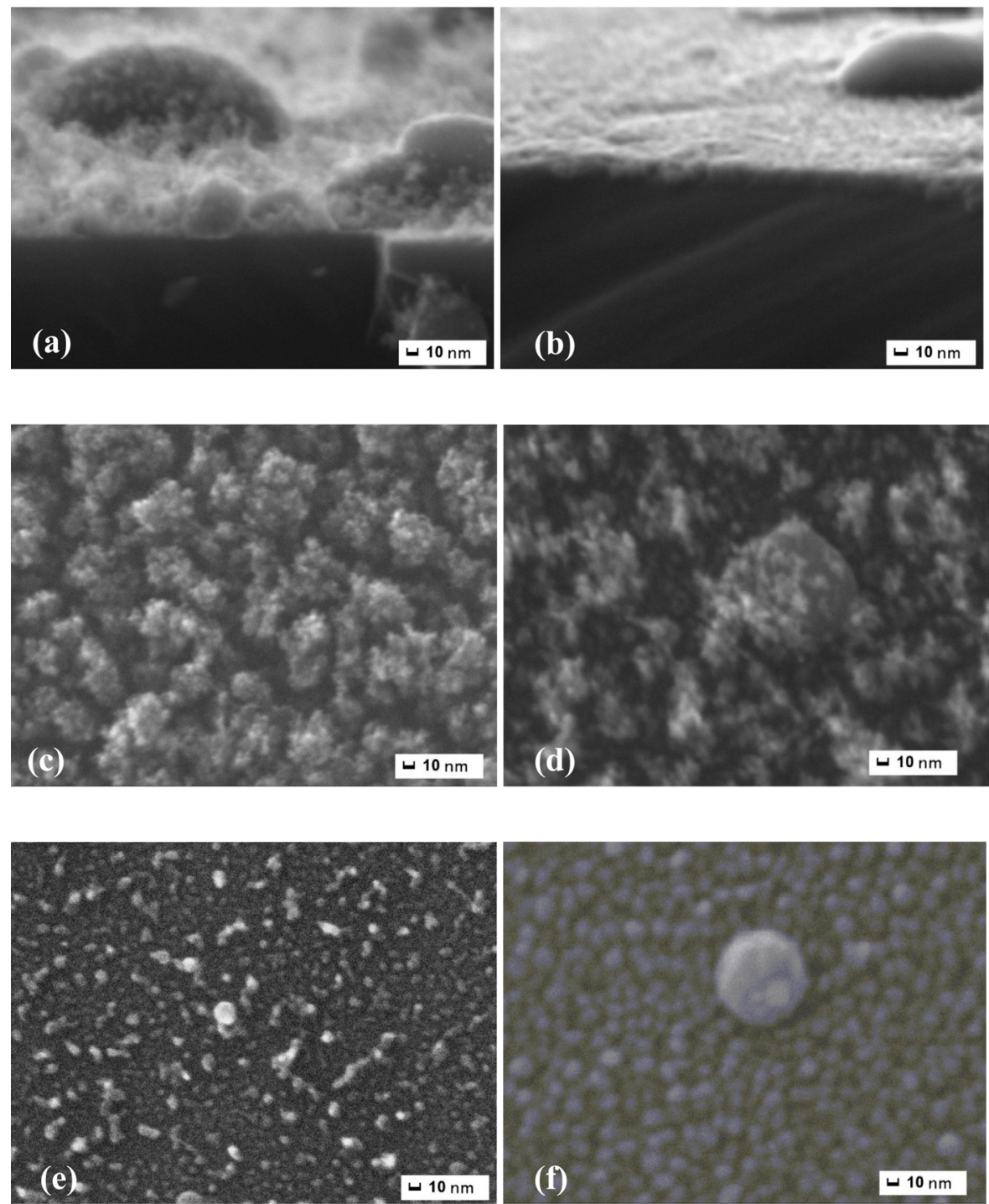

Figure 1. SEM analysis of (a and c) RT, (d) AN300, (e) AN550 and (b and f) DEP300. Substrate is silicon for all samples.

irradiated using an ABET solar simulator (AM 1.5G, $100 \mathrm{~mW} \mathrm{~cm}^{-2}$ ), and the illuminated area was $1 \mathrm{~cm}^{2}$.

IPCE spectra were recorded under a potential bias of both 1.1 and $1.5 \mathrm{~V}$ vs RHE. The steady-state photoanodic current was recorded every $10 \mathrm{~nm}$ from 330 and $600 \mathrm{~nm}$ on a PGSTAT 30 electrochemical workstation. The incident monochromatic irradiance was measured with a calibrated silicon photodiode (Centronic ASD100-7Q). IPCE was calculated according to

$$
\mathrm{IPCE}=1.24 \times 10^{-3}(\mathrm{Vm}) \frac{J_{\lambda}\left(\mu \mathrm{A} \mathrm{cm}^{-2}\right)}{\lambda(\mathrm{nm}) P_{\lambda}\left(\mathrm{Wm}^{-2}\right)}
$$

where $J_{\lambda}$ is the photocurrent density at the wavelength $\lambda$ and $P_{\lambda}$ is the incident radiant power. APCE was obtained according to IPCE/LHE, where $\operatorname{LHE}(\lambda)=\left(1-10^{-A(\lambda)}\right)$ and $A$ is the absorbance of the photoelectrode measured in transmission mode taken from the electronic spectra reported in Figure S5.

Dissolved oxygen evolved during (photo)electrochemical experiments with HTL-AN300 and AN300 was measured in a sealed cell under nitrogen atmosphere by using a polarographic sensor (Crison Oxi $45+)$ calibrated in air-saturated water by following a single point procedure. Comparative $\mathrm{O}_{2}$ evolution experiments were carried out under galvanostatic conditions (using the same current as that observed with the Fe-based thin films) by using $\mathrm{IrO}_{2} / \mathrm{FTO}$ working electrode obtained by spin-casting colloidal $\mathrm{IrO}_{2}$ nanoparticles fabricated according to Hara et al. ${ }^{21}$

Potentiostatic impedance data of photoanodes under illumination were recorded in the three-electrode configuration cell described above in $0.1 \mathrm{M} \mathrm{NaOH}$ ( $\mathrm{pH}$ 13.3). Sample data were obtained from -0.73 to $1.53 \mathrm{~V}$ vs RHE at $50 \mathrm{mV}$ intervals employing a FRA2.v10 frequency-response analyzer controlled by Nova 1.10. A $10 \mathrm{mV}$ amplitude sinusoidal perturbation (single sine) whose frequency ranged between 100000 and $0.05 \mathrm{~Hz}$ was adopted. The impedance response was fitted using ZView software with the electric equivalent reported in Figure S9.

\section{RESULTS AND DISCUSSION}

Cross-sectional SEM analysis of RT samples deposited on silicon substrates reveals a complex morphology with scattered particles of diameter in the range of about $30-300 \mathrm{~nm}$ covered by a porous structure made of smaller particles assembled in irregular wires (Figures 1a and S1 for lower magnification images). As the size of these latter particles approaches the spatial resolution of the SEM apparatus $(2 \mathrm{~nm})$, a conservative estimation for their average diameter is $5 \pm 2 \mathrm{~nm}$. Because of the very rough morphology, it is not possible to define a layer thickness, but the surface coverage appears to be complete (Figure 1c). Annealing of RT samples at $300{ }^{\circ} \mathrm{C}$ results in a 
partial collapse of the porous structure by aggregation of the smaller particles (AN300, Figure 1d), which becomes complete after annealing at $550{ }^{\circ} \mathrm{C}$ (AN550, Figure 1e). Deposition with the substrate heated at $300{ }^{\circ} \mathrm{C}$ (DEP300) results in a more compact NPs-assembled layer (Figure $1 \mathrm{~b}, \mathrm{f}$, cross-sectional and top views, respectively) similar to the structures typically obtained with lower deposition pressures. ${ }^{22}$ This effect is due to the higher mobility of the particles when they hit the heated substrate, leading to aggregation and the formation of bigger and partially fused particles, mostly of diameter $>10 \mathrm{~nm}$.

In the case of the high-pressure depositions employed here, the oxygen atmosphere reduces the kinetic energy of the ablated material, limits the plume expansion, and promotes collisions between ablated particles. This results in the formation of nanoclusters in the plume, which are cooled down by the background gas before reaching the substrate where they create a porous structure. By contrast, in lower pressure conditions, the plume expansion is less confined, the ablated material retains an higher kinetic energy, and the higher mobility allows aggregation of the particles after deposition on the substrate. The result is a more dense microstructure composed of partially fused NPs. ${ }^{23,24}$

Raman analysis (Figure 2) reveals the presence of the crystalline hematite main peaks ${ }^{25}$ at 226 and $292 \mathrm{~cm}^{-1}$ only in

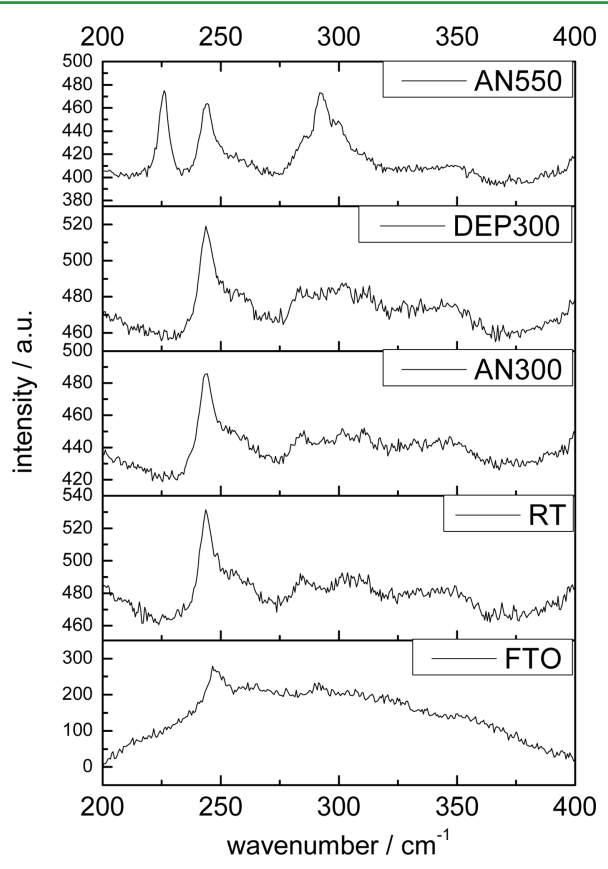

Figure 2. Raman spectra on FTO substrates.

the case of the sample annealed at $550{ }^{\circ} \mathrm{C}$. All other samples show spectra with features not clearly different from the FTO reference. This is an indication of the largely amorphous nature of the deposited material, which is known to yield better OER catalysis performance than the crystalline phase. ${ }^{26}$

XPS analysis confirms that the only oxidation state of iron is $\mathrm{Fe}(\mathrm{III})\left(\mathrm{Fe} 2 \mathrm{p}_{1 / 2}\right.$ peak $\mathrm{BE}=710.2 \pm 0.5 \mathrm{eV}$ and $\mathrm{Fe} 2 \mathrm{p}_{3 / 2}$ peak $\mathrm{BE}=723.7 \pm 0.5 \mathrm{eV}$; see Figure S2) in all samples.

From the analysis of UV-vis absorption spectra on quartz substrates (Figure 3), two important observations emerge: (i) The sample DEP300 shows a much higher absorbance in all the investigated range. (ii) The absorbance correlates with

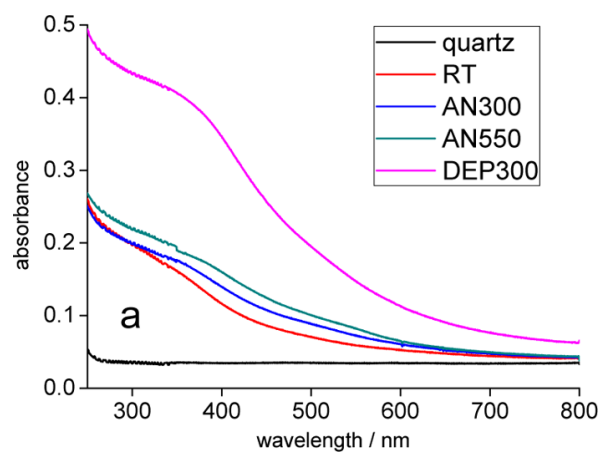

Figure 3. UV-vis absorption spectra on quartz substrates.

morphology in the direction of higher values for more compact films.

In the PLD methodology, the quantity of deposited material depends essentially on (a) the ablation rate, (b) the background gas pressure that determines the plume expansion, (c) the target-to-substrate distance, and (d) the number of laser pulses. In our case, $\mathrm{a}-\mathrm{c}$ are kept constant, and only the number of pulses is varied. The ablation rate depends on the laser fluency $\left(\mathrm{J} / \mathrm{cm}^{2}\right)$, which is constant, and on the target morphology, which is altered by the ablation process. In our system, this effect is minimized by a pattern of target motion designed to ensure homogeneous erosion of the target surface. ${ }^{20}$ In other reports by our group, where the quantity of catalyst involved is higher, the relation between number of pulses and deposited material has been verified by weighing the substrates before and after the deposition. ${ }^{27,28}$ In other papers where thin-films morphologies were investigated, we verified a reproducible relation between film thickness (and hence the quantity of material deposited) and number of pulses. ${ }^{22,29}$

Given that the quantity of deposited material at a given pressure is essentially set by the number of pulses, which is kept constant for all depositions, the difference between RT and DEP300 is all the more striking. The reason for this behavior probably lies in the different morphology of the films. DEP300 is essentially a bidimensional thin-film, although with a nanostructured surface, whereas RT exhibits a tridimensional architecture with scattered big particles and a substantial fraction of the surface composed of very small particles $(5 \pm 2$ $\mathrm{nm}$ ). In this latter case, a reasonable hypothesis is that a size effect in the form of optical quantum confinement dominates the optical properties, as reported for other materials ${ }^{30}$ and for other nanosized hematites. ${ }^{31,32}$ A recent investigation ${ }^{17}$ observed a remarkable blueshift of up to $0.3 \mathrm{eV}$ for both direct and indirect transitions when decreasing a hematite film thickness from 20 to $4 \mathrm{~nm}$, leading to an high degree of transparency in the visible region. The effect is size-dependent and ascribed by the authors to quantum confinement. It should be however recalled that also a small change in the refractive index of the catalytic film could lead to enhanced transparency. Nevertheless, the observation of a well-defined emission band at ca. $450 \mathrm{~nm}$, consistent with the band gap of the RT and AN300 thin films, is suggestive of the presence and possibly of the coexistence of quantum-optical phenomena (Figure S6). Such a feature appears to characterize only the RT and AN300 samples and is lost in the AN550 and DEP300 thin films in agreement with the collapse of the nanostructures observed by SEM micrography. Reducing hematite particle diameter to the $5 \pm 2 \mathrm{~nm}$ level could help alleviate the problem of a very short hole-diffusion length, lying in the nanometer range, ${ }^{33}$ but the 

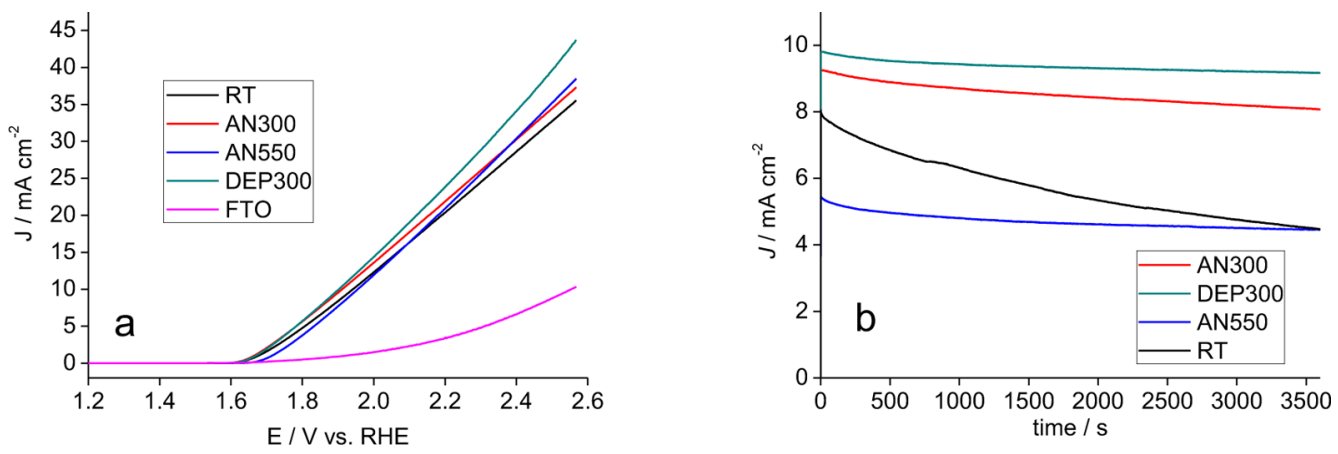

Figure 4. (a) Linear sweep voltammetry on FTO substrates and (b) chronoamperometry at $1.83 \mathrm{~V}$ vs RHE.
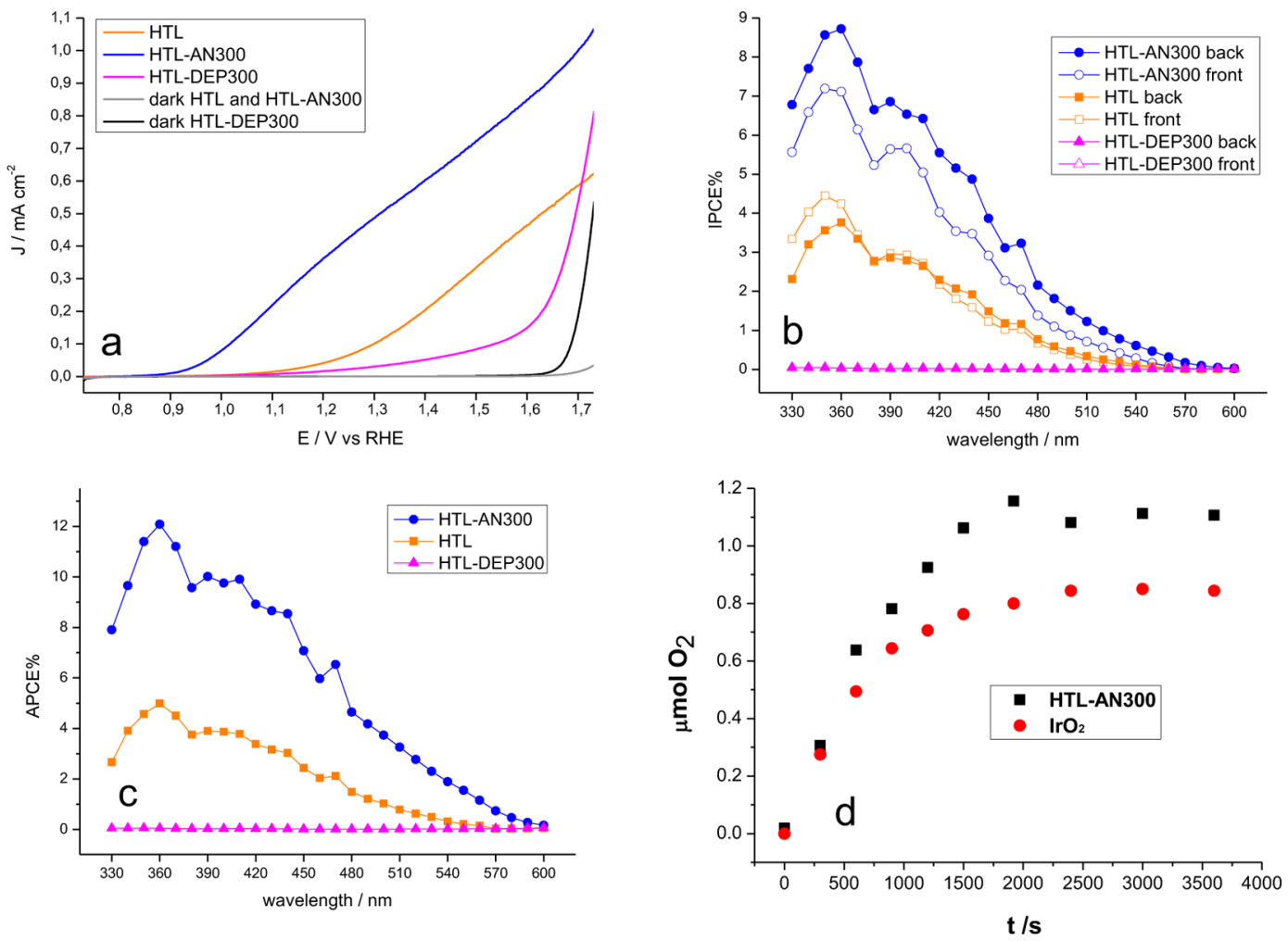

Figure 5. (a) $J-V$ curves of HTL, HTL-AN300, and HTL-DEP300 recorded under AM $1.5 \mathrm{G}$ illumination $\left(100 \mathrm{~mW} \mathrm{~cm}^{-2}\right)$ and in the dark in $0.1 \mathrm{M}$ $\mathrm{NaOH}$ ( $\mathrm{pH}$ 13.3). (b) IPCE spectra of the same samples recorded at $1.5 \mathrm{~V}$ vs RHE shining the electrodes from both the back (full symbols) and front side (empty symbols). (c) APCE spectra of the photoelectrodes reported in panel b. (d) Oxygen evolution in $0.1 \mathrm{M} \mathrm{NaOH}(\mathrm{pH} 13.3$ ) observed during constant potential photoelectrolysis with HTL-AN300 (AM $1.5 \mathrm{G}, 100 \mathrm{~mW} \mathrm{~cm}{ }^{-2}$ ) held at $1.52 \mathrm{~V}$ vs RHE (black squares) compared to the dark electrolysis with an $\mathrm{IrO}_{2} / \mathrm{FTO}$ electrode (red circles) biased at the same current $(0.48 \mathrm{~mA})$ of the hematite based photoelectrode.

resulting low absorption is an issue in the design of photoactive devices based on a single material. This effect can instead be exploited to functionalize an absorber with a transparent iron oxide OER catalyst.

$\mathrm{Fe}_{2} \mathrm{O}_{3}$ is stable in alkaline environment $(\mathrm{pH}>9.8)$, so electrochemical water oxidation experiments were performed at $\mathrm{pH} 14(1 \mathrm{M} \mathrm{NaOH})$ to ensure a well-defined electrochemistry. The oxygen evolution is expected at potential more positive than $1.23 \mathrm{~V}$ vs RHE. Figure 4a illustrates the current responses of the amorphous iron oxide films cast on FTO along with that of a bare FTO taken as a reference.

The linear scan reveals that all samples are active toward water oxidation, with both DEP300 and AN300 showing considerable electroactivity with overpotentials (taken at current density $J=0.2 \mathrm{~mA} \mathrm{~cm}{ }^{-2}$ ) of 408 and $433 \mathrm{mV}$, respectively. However, the current response of RT decreased considerably during a constant potential electrolysis experiment at $1.83 \mathrm{~V}$ vs RHE, designed to test film stability under operational conditions at moderate current densities (Figure $4 \mathrm{~b})$. The current density loss for RT is of $44 \%$, whereas DEP300 is substantially stable with only a $6 \%$ loss. AN300 and AN550 show an intermediate stability with a relative decrease of $10-11 \%$. In the case of RT, the performance degradation is irreversible and is due to detachment of active material, as observed by SEM images taken after prolonged electrolysis (Figure S3). Conversely, the initial performance of AN300 was reobserved on successive $J-V$ scans (Figure S4) after restoring the electrode by abundant rinsing with water and heating at 80 ${ }^{\circ} \mathrm{C}$ in air for $2 \mathrm{~h}$. In this case, the current decrease during electrolysis may originate by adsorption of molecular oxygen, ions, and oxidation intermediates, limiting the active surface of the electrode, rather than by mechanical detachment of a- 

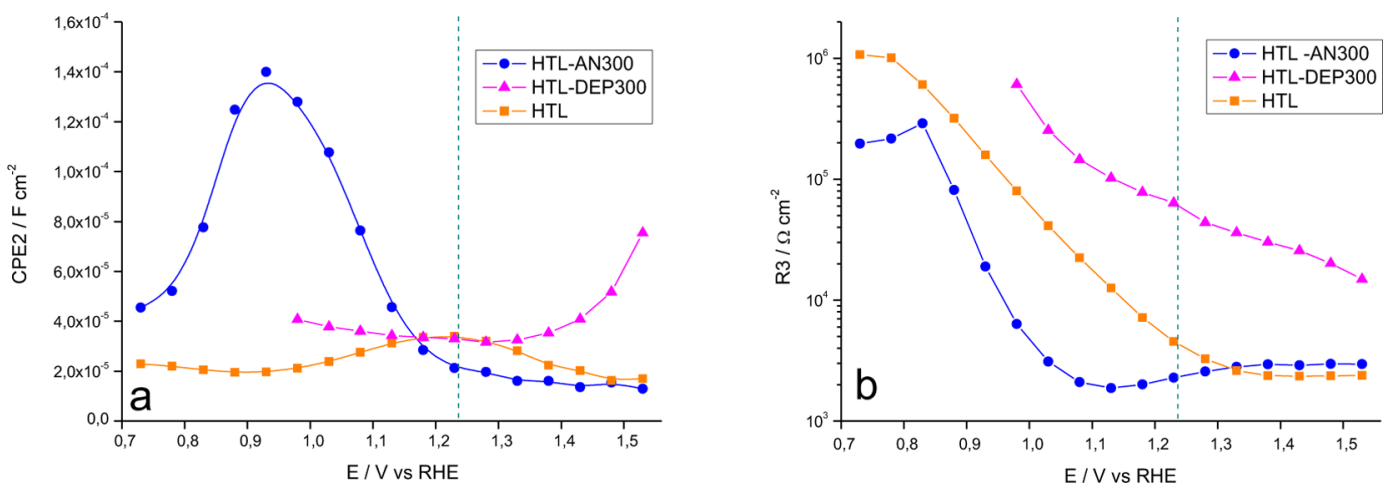

Figure 6. (a) CPE2 and (b) R3 plot of HTL, HTL-AN300, and HTL-DEP300 recorded under AM 1.5G illumination in $0.1 \mathrm{M} \mathrm{NaOH}$ (pH 13.3). The dotted vertical bar in the graphs indicates the thermodynamic potential of water oxidation.

$\mathrm{Fe}_{2} \mathrm{O}_{3}$. From a purely electrocatalytic point of view, DEP300 is the best electrode, with $J=41.2 \mathrm{~mA} \mathrm{~cm}^{-2}$ at $2.53 \mathrm{~V}$ vs RHE, whereas AN550 is limited to $36 \mathrm{~mA} \mathrm{~cm}^{-2}$ at the same potential value, confirming that the amorphous material is a better catalyst than crystalline hematite, as reported by recent literature. $^{26,34}$ To verify the application of these iron-based catalysts in photocatalytic devices, HTL electrodes prepared by spin-coating have been functionalized by PLD with AN300 and DEP300 iron-based structures (indicated as HTL-AN300 and HTL-DEP300). The current-voltage behavior of the modified HTLs, recorded by scanning the photoelectrodes under AM $1.5 \mathrm{G}$ illumination (at $100 \mathrm{~mW} \mathrm{~cm}^{-2}$ simulated sunlight) in 0.1 $\mathrm{M} \mathrm{NaOH}$ ( $\mathrm{pH}$ 13.3), reveals a significantly improved photoelectrode performance after the functionalization with AN300 (Figure 5a). The photocurrent improves along the whole potential region of interest, reaching $0.4 \mathrm{~mA} \mathrm{~cm}^{-2}$ at 1.23 $\mathrm{V}$ vs RHE with respect to ca. $0.08 \mathrm{~mA} \mathrm{~cm}^{-2}$ for the unmodified HTL at the same potential. The curve of HTL-AN300 also shows a cathodic shift of the photocurrent onset of about 200 $\mathrm{mV}$ (ca. 0.9 V vs RHE for HTL-AN300 with respect to $1.1 \mathrm{~V}$ vs RHE observed for the bare HTL film) representing, so far, one of the most prominent OEC-induced decreases in photoanodic overpotential. ${ }^{35,36}$ Differently, the functionalization of HTL with the more compact DEP300 has an adverse effect on the photocurrent generation. The "dark" catalytic effect of DEP300 is evident from the rising of the anodic discharge starting at ca. $1.6 \mathrm{~V}$ vs RHE, but the photocurrent generated at lower applied bias is largely inferior to that observed with the unmodified HTL, suggesting a significant degree of recombination or the blocking of the hole transfer from the underlying HTL, where photocharge is generated, to the electrolyte. IPCE spectra recorded at $1.5 \mathrm{~V}$ vs RHE are consistent with the $J-V$ response discussed above, showing better charge generation and transfer properties for the AN300 modified photoelectrode (Figure 5b). Given the nanometer thicknesses of the photoelectrode and of the catalytic overlayer, no large differences were observed by changing the illumination mode from back (contact side) to front (electrolyte side), in agreement with the absorption profile of both the unmodified and modified samples (Figure S5), ruling out a parasitic effect of the catalysts on the absorption capability of the semiconductor. IPCE spectra collected in back and front mode are indeed nearly superimposable, with only a slight improvement in the absolute values of photon to electron conversion observed in back illumination mode, consistent with the shorter electron collection path. The photoaction spectra of HTL-AN300 recorded at $1.1 \mathrm{~V}$ vs RHE (Figure S7), where no photocurrent is detected for the unmodified HTL sample, display the same shape of the IPCE spectra recorded at $1.5 \mathrm{~V}$ vs RHE for both HTL and HTL-AN300, suggesting that the charge generation involves essentially the underlying crystalline hematite and that the AN300 catalyst improves the photoresponse of the combined electrode by participating essentially to the secondary hole scavenging events that follow the primary charge generation within the crystalline hematite film. APCE spectra (Figure 5c) corroborate the piece of evidence gained from the IPCE measurements, showing that the HTL-AN300 photoelectrode is intrinsically able to generate and separate photocharge with an efficiency which is at least twice that of the unmodified HTL.

Amperometric oxygen measurements in solution confirm that both the AN300 catalyst (Figure S8) and HTL-AN300 (Figure 5d) are able to evolve oxygen at a rate which is comparable to that of a transparent $\mathrm{IrO}_{2} /$ FTO electrode biased at the same anodic current.

Electrochemical impedance spectroscopy (EIS) data allowed us to obtain more precise information about the nature of the charge transfer processes and were interpreted with the simplified equivalent circuit reported in previous contributions ${ }^{18,37,38}$ and in Figure S9, comprising a nested mesh describing both the charge transfer across the depletion layer (R2-CPE1) and the Helmholtz layer (R3-CPE2). The charge transfer resistance (R3) associated with the trapping of holes in surface states is the dominating resistive component in the interfacial dynamics of these type of hematite photoelectrodes, describing well the $J-V$ characteristic for both unmodified and AN300 modified films (Figure S10). The capacitance associated with such resistance (CPE2) follows in both HTL and HTLAN300 a bell-shaped distribution typical of the trapping of photoholes in surface states (Figure 6a), leading to the generation of highly valent reactive states responsible for the formation of $\mathrm{Fe}(\mathrm{IV})=\mathrm{O}$ type reactive species, which are considered the first key intermediates for the oxygen evolution reaction. ${ }^{39}$ The trapped photohole capacitance undergoes indeed a negative shift and a significant enhancement in the presence of the porous AN300 catalyst $\left(1.56 \times 10^{-5}\right.$ and $4.56 \times$ $10^{-5} \mathrm{~F}$ for the integrated values of HTL and HTL-AN300 respectively), justifying the improved performances of the latter. The formation of oxidized catalyst states in the porous layer must be compensated by the exchange of ions (namely, $\mathrm{OH}^{-}$and $\mathrm{H}^{+} / \mathrm{Na}^{+}$) with the electrolyte probably resulting in the formation of an adaptive junction with the semiconductor, $^{13}$ where the Fermi level of the photoactive semiconductor is essentially unpinned from that of the 

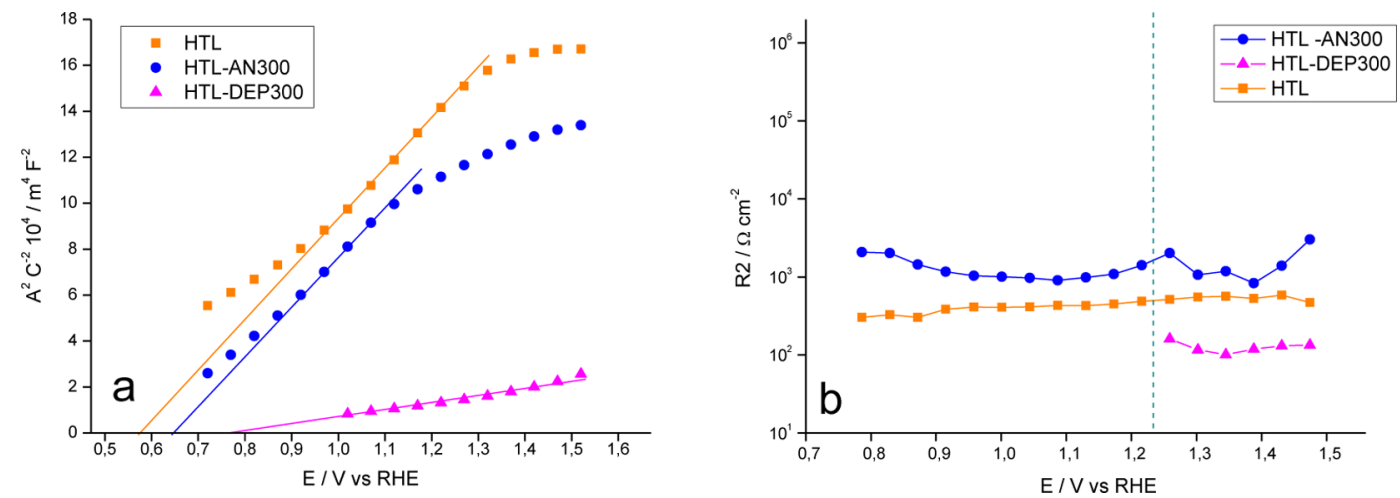

Figure 7. (a) Mott-Schottky plot recorded at $100 \mathrm{~Hz}$ under AM 1.5 illumination in $0.1 \mathrm{M} \mathrm{NaOH}$ (pH 13.3) for HTL, HTL-AN300, and HTLDEP300. (b) R2 plot of the same samples.

amorphous catalyst present in the form of highly nanostructured or discontinuous islands. At present, our attempts to establish an ohmic contact with the catalyst by evaporating a gold contact in order to independently probe its Fermi level, as described in ref 13 , failed due to electrical shorting. This may be due to the exceedingly thin active layers and to the porous morphology of the outer catalytic layer resulting in the direct contact of gold with either the crystalline semiconductor or FTO. Nevertheless, the trapping of holes corresponding to formation and stabilization of $\mathrm{Fe}$ (IV) species, revealed clearly by EIS, should automatically imply a variation of the Fermi level of the catalyst that is able to move independently from that of the semiconductor. APCE spectra (Figure 5c) rule out a role of light absorption in explaining the improved performance of the HTL-AN300, consistent with EIS result being able to separate photocharge with a maximum internal efficiency that is about twice that of the unmodified HTL. It should be noted that the charge transfer resistance shows a broad minimum correspondening to the capacitance maximum of the HTLAN300 (Figure 6b), corroborating the correlation between improved surface trap capacitance and reduced charge transfer resistance of the interface. By contrast, the compact catalytic film (DEP300) is seemingly unable to trap holes, with the interfacial capacitance being essentially constant and at low values (ca. $3.5 \times 10^{-5} \mathrm{~F} \mathrm{~cm}^{-2}$ ) within the whole potential interval where photocurrent is observed, only showing a sizable increase at potentials corresponding to the dark anodic discharge (Figure 6a) where the Fermi level under potentiostatic control intercepts directly the electrochemically active sites of the DEP300 film. The reduced performance of the compact modified HTL-DEP300 junction might be due to both the compact morphology of DEP300, offering a smaller number of reactive sites where photoholes could trap, and to the lower permeability of this compact layer to the electrolyte, which does not allow charge to compensate as easily as in the AN300 layer the trapping of photoholes with the movement of anions to and from the electrolyte. Consistent with such hypothesis, the charge transfer resistance is significantly higher than that in both HTL and HTL-AN300 (Figure 6b), steadily decreasing in an almost linear fashion with the increasing potential, instead of sharply dropping as observed in the latter cases.

Finally, the $100 \mathrm{~Hz}$ Mott-Schottky plots show a linear behavior of the $1 / C^{2}$ versus applied potential, in agreement with the formation of a depletion layer (Figure 7a). The donor density (ca. $3 \times 10^{17} \mathrm{~cm}^{-3}$ ) for the HTL and HTL-AN300 agree with previously found values for similar type of electrodes fabricated by our groups. ${ }^{18}$ By contrast, although the HTLDEP300 shows a linear behavior as well, a 10-fold increased donor density $\left(2 \times 10^{18} \mathrm{~cm}^{-3}\right)$ is found, suggesting that the depletion layer may extend into the amorphous compact layer having a larger number of defects which contribute to boosting the overall donor density. This effect could be useful to improve the overall n-type conductivity of the thin films; the resistance associated with the depletion layer (R2) is indeed smaller in the HTL-DEP300 with respect to the other two electrode types (Figure $7 \mathrm{~b}$ ) but will be quite irrelevant in determining the performance of combined films having an overall thickness of less than $50 \mathrm{~nm}$. Most importantly, the involvement of the space charge layer within the DEP300 is consistent with the fact that Fermi level variations within the catalytic DEP300 film occur by acting on the width of the depletion layer rather than by charge compensation with the electrolyte and that the Fermi levels of the crystalline photoelectrode and of the catalyst are not able to move independently of each other. Photoholes will thus have to travel from the crystalline layer through the defective amorphous DEP300 layer, being exposed to recombination reactions, before reaching the interface with the electrolyte where scavenging, leading to oxygen evolution, could finally occur. This explains the lack of a distinct hole-trapping capacitance peak as well as the low capacitance values and the high charge transfer resistance, consistent with DEP300 behaving, in practice, as an intermediate hole-blocking interface, resulting in a large decrease of the photocurrent with respect of that of the unmodified sample. Only the dark electrochemical discharge will be evident in the combined HTL-DEP300 electrode, as indeed experimentally observed, when the applied potential, moving further to anodic values, directly intercepts the redox active sites of the catalyst.

\section{CONCLUSIONS}

PLD, an easily scalable fabrication technique already applied in a variety of industrial processes, ${ }^{40}$ proves to be a versatile method to control morphology at the nanometric scale, thus allowing a systematic investigation of morphological and electronic effects on the properties of functional materials. In this work we have designed and synthesized by PLD a porous amorphous $\mathrm{Fe}_{2} \mathrm{O}_{3}$ OEC based on a nanoparticle-assembled coating morphology. This coating is found to be highly transparent in the visible range possibly due to a quantum confinement effect induced by the size of the nanostructures in the order of few nanometers and to retain the previously 
documented catalytic activity. Unfortunately, the catalytic layer is unstable under electrocatalytic conditions in the presence of moderate current densities $\left(<10 \mathrm{~mA} / \mathrm{cm}^{2}\right)$. A simple lowtemperature thermal treatment $\left(300{ }^{\circ} \mathrm{C}\right)$ was found to improve film stability enough for its successful application as catalytic overcoating for hematite thin film photoanodes, resulting in a dramatic decrease (of about $200 \mathrm{mV}$ ) of the photocurrent onset and a remarkable 5-fold increase in the photocurrents detected in a photoelectrochemical cell. We believe that this is among the most prominent catalytic effects obtained from the coupling of a surface OEC with a thin layer absorber. By contrast, functionalization of the hematite photoanodes with a more compact catalyst morphology, exhibiting better electrocatalytic properties, is detrimental to photocurrent generation. In our case, $J-V$ curves under illumination, IPCE analysis, and EIS point to the nature of the absorber/catalyst junction rather than to the parasitic light blocking as the dominant factor in determining the PEC performance. Indeed, APCE and EIS data are consistent with a much better charge separation in the porous morphology that may stem from the formation of an adaptive junction allowing for the efficient storage of photogenerated holes within the porous catalyst. By contrast, the compact overlayer probably forms a buried junction, inhibiting hole transfer to the electrolyte. Regarding parasitic light absorption, while not critical in this case, likely due to the low thickness of the coatings ( $<20 \mathrm{~nm}$ for the compact film), the high transmittance, and solvent permeability of the porous morphology opens up the possibility of employing higher mass loadings of catalyst without loss of performance. This would be especially beneficial for earth-abundant, cheap, and scalable materials such as iron oxides.

Further work will be aimed at solving the stability problem of the most-transparent RT structures without resorting to the postdeposition thermal treatment by exploring deposition temperatures lower than $300{ }^{\circ} \mathrm{C}$ and at extending this methodology to more intrinsically active species such as bifunctional mixed-metal oxides.

\section{ASSOCIATED CONTENT}

\section{S Supporting Information}

The Supporting Information is available free of charge on the ACS Publications website at DOI: 10.1021/acsami.6b05135.

Additional SEM images, XPS spectra, additional $J-V$ curves, UV-vis spectra, emission spectra, IPCE spectra, oxygen evolution measurements under electrochemical conditions, and EIS data including the equivalent circuit used for fitting (PDF)

\section{AUTHOR INFORMATION}

\section{Corresponding Author}

*E-mail: michele.orlandi@unitn.it.

\section{Funding}

The research activity is partially supported by the PAT (Provincia Autonoma di Trento) project ENAM in cooperation with Istituto MCB of CNR (Italy). FIRB Project Nanosolar (UNIFE) and the European Union's Horizon 2020 Research and Innovation Programme under the Marie Skłodowska-Curie Grant Agreement No. 705723 (S.B.) is gratefully acknowledged for funding (UNIFE).

\section{Notes}

The authors declare no competing financial interest.

\section{ACKNOWLEDGMENTS}

We gratefully acknowledge Claudio Cestari and Luigino Vivaldi for technical help. We are thankful to Teodoro Klaser for useful discussions.

\section{ABBREVIATIONS}

PEC, photoelectrochemical cell

OEC, oxygen evolution catalyst

PLD, pulsed-laser deposition

EIS, electrochemical impedance spectroscopy

IPCE, incident photon-to-current efficiency

APCE, absorbed photon-to-current efficiency

\section{REFERENCES}

(1) Rüttinger, W.; Dismukes, G. C. Synthetic Water-Oxidation Catalysts for Artificial Photosynthetic Water Oxidation. Chem. Rev. 1997, 97, 1-24.

(2) Lewis, N. S. Light Work with Water. Nature 2001, 414, 589-590.

(3) Kamat, P. V.; Bisquert, J. Solar Fuels. Photocatalytic Hydrogen Generation. J. Phys. Chem. C 2013, 117, 14873-14875.

(4) Youngblood, W. J.; Lee, S.-H. A.; Kobayashi, Y.; HernandezPagan, E. A.; Hoertz, P. G.; Moore, T. A.; Moore, A. L.; Gust, D.; Mallouk, T. E. Photoassisted Overall Water Splitting in a Visible LightAbsorbing Dye-Sensitized Photoelectrochemical Cell. J. Am. Chem. Soc. 2009, 131, 926-927.

(5) Reece, S. Y.; Hamel, J. A.; Sung, K.; Jarvi, T. D.; Esswein, A. J.; Pijpers, J. J. H.; Nocera, D. G. Wireless Solar Water Splitting Using Silicon-Based Semiconductors and Earth-Abundant Catalysts. Science 2011, 334, 645-648.

(6) Cristino, V.; Berardi, S.; Caramori, S.; Argazzi, R.; Carli, S.; Meda, L.; Tacca, A.; Bignozzi, C. A. Efficient Solar Water Oxidation using Photovoltaic Devices Functionalized with Earth-abundant Oxygen Evolving Catalysts. Phys. Chem. Chem. Phys. 2013, 15, 13083-92.

(7) Orlandi, M.; Argazzi, R.; Sartorel, A.; Carraro, M.; Scorrano, G.; Bonchio, M.; Scandola, F. Ruthenium Polyoxometalate Water Splitting Catalyst: Very Fast Hole Scavenging from Photogenerated Oxidants. Chem. Commun. 2010, 46, 3152-4.

(8) Klahr, B.; Gimenez, S.; Fabregat-Santiago, F.; Bisquert, J.; Hamann, T. W. Photoelectrochemical and Impedance Spectroscopic Investigation of Water Oxidation with "Co-Pi"-coated Hematite Electrodes. J. Am. Chem. Soc. 2012, 134, 16693-700.

(9) Sun, J.; Zhong, D. K.; Gamelin, D. R. Composite Photoanodes for Photoelectrochemical Solar Water Splitting. Energy Environ. Sci. 2010, 3, 1252-1261.

(10) Sartorel, A.; Bonchio, M.; Campagna, S.; Scandola, F. Tetrametallic Molecular Catalysts for Photochemical Water Oxidation. Chem. Soc. Rev. 2013, 42, 2262-2280.

(11) Ye, H.; Park, H. S.; Bard, A. J. Screening of Electrocatalysts for Photoelectrochemical Water Oxidation on W-Doped BiVO4 Photocatalysts by Scanning Electrochemical Microscopy. J. Phys. Chem. C 2011, 115, 12464-12470.

(12) McKone, J. R.; Lewis, N. S.; Gray, H. B. Will Solar-Driven Water-Splitting Devices See the Light of Day? Chem. Mater. 2014, 26, 407-414.

(13) Lin, F.; Boettcher, S. W. Adaptive Semiconductor/Electrocatalyst Junctions in Water-Splitting Photoanodes. Nat. Mater. 2013, 13, $81-86$.

(14) Trotochaud, L.; Mills, T. J.; Boettcher, S. W. An Optocatalytic Model for Semiconductor-Catalyst Water-Splitting Photoelectrodes Based on In Situ Optical Measurements on Operational Catalysts. J. Phys. Chem. Lett. 2013, 4, 931-935.

(15) Popczun, E. J.; McKone, J. R.; Read, C. G.; Biacchi, A. J.; Wiltrout, A. M.; Lewis, N. S.; Schaak, R. E. Nanostructured Nickel Phosphide as an Electrocatalyst for the Hydrogen Evolution Reaction. J. Am. Chem. Soc. 2013, 135, 9267-9270. 
(16) McKone, J. R.; Sadtler, B. F.; Werlang, C. A.; Lewis, N. S.; Gray, H. B. Ni-Mo Nanopowders for Efficient Electrochemical Hydrogen Evolution. ACS Catal. 2013, 3, 166-169.

(17) Fondell, M.; Jacobsson, T. J.; Boman, M.; Edvinsson, T. Optical Quantum Confinement in Low Dimensional Hematite. J. Mater. Chem. A 2014, 2, 3352.

(18) Dalle Carbonare, N.; Carli, S.; Argazzi, R.; Orlandi, M.; Bazzanella, N.; Miotello, A.; Caramori, S.; Bignozzi, C. A. Improvement of the Electron Collection Efficiency in Porous Hematite Using a Thin Iron Oxide Underlayer: Towards Efficient All-Iron Based Photoelectrodes. Phys. Chem. Chem. Phys. 2015, 17, 29661-29670.

(19) Vesborg, P. C. K.; Jaramillo, T. F. Addressing the Terawatt Challenge: Scalability in the Supply of Chemical Elements for Renewable Energy. RSC Adv. 2012, 2, 7933.

(20) Bonelli, M.; Cestari, C.; Miotello, A. Pulsed Laser Deposition Apparatus for Applied Research. Meas. Sci. Technol. 1999, 10, 27.

(21) Hara, M.; Waraksa, C. C.; Lean, J. T.; Lewis, B. A.; Mallouk, T. E. Photocatalytic Water Oxidation in a Buffered Tris $\left(2,2^{\prime}\right.$-bipyridyl $)$ ruthenium Complex-Colloidal IrO2 System. J. Phys. Chem. A 2000, 104, 5275-5280.

(22) Orlandi, M.; Caramori, S.; Ronconi, F.; Bignozzi, C. A.; El Koura, Z.; Bazzanella, N.; Meda, L.; Miotello, A. Pulsed-Laser Deposition of Nanostructured Iron Oxide Catalysts for Efficient Water Oxidation. ACS Appl. Mater. Interfaces 2014, 6, 6186-6190.

(23) Infortuna, A.; Harvey, A. S.; Gauckler, L. J. Microstructures of CGO and YSZ Thin Films by Pulsed Laser Deposition. Adv. Funct. Mater. 2008, 18, 127-135.

(24) Petrov, I.; Barna, P. B.; Hultman, L.; Greene, J. E. Microstructural Evolution During Film Growth. J. Vac. Sci. Technol., A 2003, 21, S117-S128.

(25) Jubb, A. M.; Allen, H. C. Vibrational Spectroscopic Characterization of Hematite, Maghemite, and Magnetite Thin Films Produced by Vapor Deposition. ACS Appl. Mater. Interfaces 2010, 2, 2804-2812.

(26) Smith, R. D.; Prevot, M. S.; Fagan, R. D.; Zhang, Z.; Sedach, P. A.; Siu, M. K.; Trudel, S.; Berlinguette, C. P. Photochemical Route for Accessing Amorphous Metal Oxide Materials for Water Oxidation Catalysis. Science 2013, 340, 60-3.

(27) Edla, R.; Patel, N.; Orlandi, M.; Bazzanella, N.; Bello, V.; Maurizio, C.; Mattei, G.; Mazzoldi, P.; Miotello, A. Highly PhotoCatalytically Active Hierarchical 3D Porous/Urchin Nanostructured Co3O4 Coating Synthesized by Pulsed Laser Deposition. Appl. Catal, B 2015, 166-167, 475-484.

(28) Patel, N.; Fernandes, R.; Guella, G.; Miotello, A. NanoparticleAssembled Co-B Thin Film for the Hydrolysis of Ammonia Borane: a Highly Active Catalyst for Hydrogen Production. Appl. Catal., B 2010, 95, 137-143.

(29) Schenato, M.; Ricardo, C. L. A.; Scardi, P.; Edla, R.; Miotello, A.; Orlandi, M.; Morrish, R. Effect of Annealing and Nanostructuring on Pulsed Laser Deposited WS2 for HER Catalysis. Appl. Catal., A 2016, $510,156-160$.

(30) Raymand, D.; Jacobsson, T. J.; Hermansson, K.; Edvinsson, T. Investigation of Vibrational Modes and Phonon Density of States in ZnO Quantum Dots. J. Phys. Chem. C 2012, 116, 6893-6901.

(31) Sivula, K.; Zboril, R.; Le Formal, F.; Robert, R.; Weidenkaff, A.; Tucek, J.; Frydrych, J.; Grätzel, M. Photoelectrochemical Water Splitting with Mesoporous Hematite Prepared by a Solution-Based Colloidal Approach. J. Am. Chem. Soc. 2010, 132, 7436-7444.

(32) Vayssieres, L.; Sathe, C.; Butorin, S. M.; Shuh, D. K.; Nordgren, J.; Guo, J. One-Dimensional Quantum-Confinement Effect in $\alpha$ Fe2O3 Ultrafine Nanorod Arrays. Adv. Mater. 2005, 17, 2320-2323.

(33) Klahr, B. M.; Martinson, A. B.; Hamann, T. W. Photoelectrochemical Investigation of Ultrathin Film Iron Oxide Solar Cells Prepared by Atomic Layer Deposition. Langmuir 2011, 27, 461-8.

(34) Smith, R. D.; Prevot, M. S.; Fagan, R. D.; Trudel, S.; Berlinguette, C. P. Water Oxidation Catalysis: Electrocatalytic Response to Metal Stoichiometry in Amorphous Metal Oxide Films Containing Iron, Cobalt, and Nickel. J. Am. Chem. Soc. 2013, 135, 11580-6.
(35) Tilley, S. D.; Cornuz, M.; Sivula, K.; Gratzel, M. Light-induced Water Splitting with Hematite: Improved Nanostructure and Iridium Oxide Catalysis. Angew. Chem., Int. Ed. 2010, 49, 6405-8.

(36) Young, K. M. H.; Hamann, T. W. Enhanced Photocatalytic Water Oxidation Efficiency with $\mathrm{Ni}(\mathrm{OH}) 2$ Catalysts Deposited on $\alpha$ $\mathrm{Fe} 2 \mathrm{O} 3$ via ALD. Chem. Commun. 2014, 50, 8727-8730.

(37) Klahr, B.; Gimenez, S.; Fabregat-Santiago, F.; Hamann, T.; Bisquert, J. Water Oxidation at Hematite Photoelectrodes: The Role of Surface States. J. Am. Chem. Soc. 2012, 134, 4294-4302.

(38) Monllor-Satoca, D.; Bartsch, M.; Fabrega, C.; Genc, A.; Reinhard, S.; Andreu, T.; Arbiol, J.; Niederberger, M.; Morante, J. R. What Do You Do, Titanium? Insight Into the Role of Titanium Oxide as a Water Oxidation Promoter in Hematite-Based Photoanodes. Energy Environ. Sci. 2015, 8, 3242-3254.

(39) Klahr, B.; Gimenez, S.; Fabregat-Santiago, F.; Bisquert, J.; Hamann, T. W. Electrochemical and Photoelectrochemical Investigation of Water Oxidation with Hematite Electrodes. Energy Environ. Sci. 2012, 5, 7626-7636.

(40) Gower, M. C. Industrial Applications of Laser Micromachining. Opt. Express 2000, 7, 56-67. 